\title{
An Interdisciplinary Approach For Investigating an Accident Originating From Leak- age In A Gasketed Bolted Joint
}

\author{
Michele Compare
}

Aramis s.r.l., Milano, Italy. E-mail: michele.compare@aramis3d.com

Energy Department, Politecnico di Milano, Italy.

\section{Enrico Zio}

Energy Department, Politecnico di Milano, Italy.E-mail: enrico.zio@polimi.it

Aramis s.r.l., Milano, Italy

MINES ParisTech, PSL Research University, CRC, Sophia Antipolis, France

Eminent Scholar, Department of Nuclear Engineering, College of Engineering, Kyung Hee University,

Republic of Korea

Piero Pantaleone

Tekrevolution, Roma, Italy.E-mail: p.pantaleone@tekrevolution.it

Raffaele Lardani

FENICE S.p.A., Rivoli TO, Italy.E-mail: raffaele.lardani@edison.it

Dario Palumbo

FENICE S.p.A., Rivoli TO, Italy.E-mail: Dario.PALUMBO@edison.it

We investigate an accident originating from a leak of hydrochloric acid through a gasketed bolted joint of a measuring equipment operating in a Waste Water Treatment System (WWTS). In spite of the simplicity of the failed component and of the fact that the WWTS is not classified as safety critical, the investigation requires corroborating the traditional techniques of risk analysis such as FMECA, Bow-Tie, etc., with non-standard, interdisciplinary techniques such as structural reliability and task analysis. Moreover, the identification of safety barriers for risk reduction requires applying refined modeling approaches, such as atmospheric dispersion modeling and human reliability modeling.

Keywords: Accident investigation, Gasketed Bolted Joint, Hydrochloric acid leakage, Bow-Tie, FMECA, structural reliability, task analysis.

\section{Introduction}

Accident investigation aims at identifying the sequence of events that have occurred in an accident and the related causal factors (DOE (1999); CCPS (1992)). Well-established methods exist, which help building an interpretation of the occurred accident and, on this basis, suggesting risk reduction measures for prevention and mitigation of future accidents (Sklet (2004)). In this work, we report the investigation of an accident originated from a leak of hydrochloric acid through a Gasketed Bolted Joint (GBJ) of a measuring equipment operating in a Waste Water Treatment System (WWTS). The released acid did not result in safety issues, but it affected semi-finished and finished capital parts present in the adjacent plant of an aeronautic industry served by the WWTS, with economic losses and liability concerns. In spite of the simplicity of the failed component and the fact that the WWTS is not classified as safety critical, the investigation required corroborating the traditional techniques of risk analysis such as Failure Mode, Effects and Criticality Analysis (FMECA), Bow-Tie, etc., with non-standard, interdisciplinary techniques such as structural reliability and task analysis. Moreover, the identification of safety barriers for risk reduction required applying refined modeling approaches, such as material analysis, structural reliabilty, atmospheric dispersion modeling and human reliability modeling.

The remainder of the paper is as follows. Section 2 details the accident. Section 3 shows the procedure performed to investigate the accident. Finally, Section 4 concludes the work.

\section{Proceedings of the 30th European Safety and Reliability Conference and}




\section{Description of the accident}

On June $17^{\text {th }} 2018$ at $07.53 \mathrm{am}$, the Head of the Operational Unit (HOU) was notified by an operator, alerted by the surveillance service, of a leakage of a chimical from the WWTS. At 08.07 am, the HOU alerted the internal fire fighters and, then, at 08.13 am informed the contact persons of the plants served by the WWTS about the ongoing emergency.

At $08.45 \mathrm{am}$, the emergency team entered the WWTS area and identified a hydrochloric acid leakage from a damaged GBJ underneath the acid storage tank. To stop the leakage, they shut off the upstream manual valve.

The emergency team noticed that the leakage was contained, and most of the lost acid had conveyed onto a spill basin underneath the storage tanks. Thus, they proceeded with transfering the liquid to the WWTS tanks, by means of delivery pumps. Upon completion of the cleaning activities of the area surrounding the tanks, an inspection tour of the departments neighboring the WWTS area was performed and, at $10.00 \mathrm{am}$, the emergency inside the WWTS ceased.

The released acid did not result in safety issues, but it affected semi-finished and finished capital parts present in the adjacent plant of an aeronautic industry served by the WWTS, with economic losses and liability concerns.

\section{Procedure for accident investigation}

The following procedure was implemented to identify the sequence of events occurred in the accident detailed above, together with the related causal factors and the possible improvements to the WWTS and its operation for prevention and mitigation of future accidents.

- Preliminary analysis. Given that the accident originated from a failure of the GBJ, the first step of the investigation aimed at analyzing the physical and chemical properties of the GBJ constituent parts to identify the failure causes.

- Review of the existing FMECA, with the twofold objective of checking how the leakage had been considered among the GBJ failure modes and, more generally, validating its outcomes that the WWTS does not contain critical components.

- Degradation Mode and Effect Analysis (DMEA), aimed at integrating the available analysts knowledge with the analysis of the technical-scientific literature to identify the most relevant degradation mechanisms affecting the components of the GBJ.

- Task Analysis (TA), aimed at analyzing the actions performed by the operators on the GBJ to identify their possible contri- butions to the accident.

- Structural reliability model, to estimate the GBJ leakage probability over time.

- Selection of safety barriers, to reduce the risk of future GBJ failure.

The procedure steps are detailed in the Subsections below.

\subsection{Preliminary analysis}

The failed component was a Commercial OffThe-Shelf (COTS) six screw GBJ. From its visual analysis, it apppeared evident that the loss of tightening was due to the failure of a screw, found withouth the head. This is shown in Figure 1 together with its two nearest screws. Then, the following analyses were performed to get insights about the occurred failure:

- Thorough visual examination;

- Stereoscopic examination;

- Fractographic examination with scanning electron microscope;

- Metallographic examination;

- Material analysis:

(i) Chemical analysis;

(ii) Mechanical properties;

(iii) Microstructural analysis.

The main outcomes are summarized as follows.

- The screw material is an austenitic stainless steel similar to the AISI 304 grade indicated on the failed GBJ nameplate, but further linked to copper. This makes the screw steel more similar to AISI 316 than to AISI 304.

- Screw A (Figure 1): found without the head, it had a three-zone fracture:

(i) The crack was triggered by a strong deformation and/or incipient break under the head, caused by a twisting moment; in fact, this screw was slightly longer and a little stricter than the other two, due to the resulting plastic deformation.

(ii) The incipient rupture initially propagated with a morphology of mixed fracture (i.e., mechanical and stress corrosion cracking).

(iii) The final part of the fracture was characterized by stress corrosion cracking.

- Screw B (Figure 1): although it looked intact at a first visual inspection, it actually had a torsional fracture underhead (helicoidal progression) and stresscracking in all the positions that have undergone a cold work hardening. Screw $\mathrm{B}$ was longer than screw $\mathrm{C}$, but shorter 
than screw A. Also this screw had the thread strongly damaged by the leakage of the acid at the same distance from the head as in screw A.

- Screw C (Figure 1): this was undeformed, with stress corrosion cracking affecting a small area.

\subsection{Review of the FMECA}

A FMECA study of the entire WWTS had been performed before the accident, according to standard ISO-60812 (2006) and using the risk matrix in Figure 2. This study included leakage among the failure modes of the GBJ, whose likelihood was estimated as low. This judgment, although reasonable to FMECA expert analysts, was questioned by the occurrence of the leakage. For this, we developed a structural reliability model for its validation (see Subsection 3.5).

With respect to the consequences of the leakage, coherently with the reference standard, the FMECA considered the propagation of the effects of the failure mode up to the WWTS level. The effects were judged as negligible. This result is partially in agreement with the occurred failure scenario: the system operability was not seriously weakened by the leakage, and neither safety issue for operators nor damages to the environment were experienced. However, the FMECA did not consider the possible damages to the properties outside the plant, which were not known at the time the FMECA was conducted. This required updating the FMECA study.

More generraly, no critical components was identified by the review of the FMECA: the simplicity of the system, its redundancies, the favorable environmental and operating conditions (i.e., sporadic presence of people, ventilated place, low pressures, etc.) always avoid major consequences in correspondence of non negligible occurrence probability values (Figure 2).

\subsection{DMEA}

A careful literature review was conducted to identify the most relevant degradation mechanisms

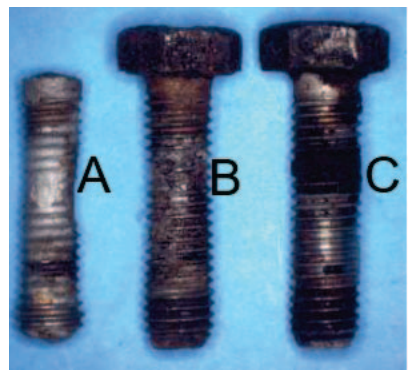

Fig. 1.: Analyzed screws

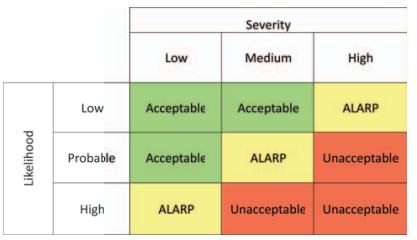

Fig. 2.: Risk matrix

affecting the components of the GBJ (Bickford (1995); European Sealing Association (1998); PCC-ASME (2000); Abid and Nash (2006); Khan et al. (2017)). Among them, the following two were identified as compatible with the outcomes of the preliminary analysis (Subsection 3.1):

- Tightening sequence is critical for joint reliability: wrong sequences entail asymmetrical bolt preloads and, thus, different stress conditions. In particular, the most stressed part is the screw underhead (Figure 3), where microcracks can originate.

- Interstitial corrosion: the water trapped under the head (e.g., due to humidity) will run out of oxygen, which migrates towards the metal while not replenished. The water, then, becomes acidic and the crack under the head (originated by the mechanism described in the previous bullet) becomes more anodic than in the adjacent regions, with consequent corrosion (Figure 4).

\subsection{Task Analysis}

The worksheet in Figure 5 was used to analyze the tasks performed by the operators on the GBJ, with the aim of identifying their contributions to risk (for details, see for example references HSE (2020); Kirwan and Ainsworth (1992)).

The outcomes of the TA confirmed that asymmetries may be introduced in the loads on the

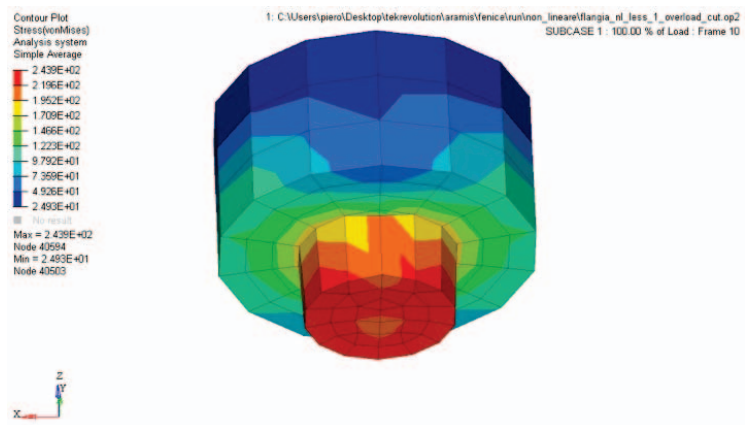

Fig. 3.: Effect of asymmetrical preload: Von Mises stress on screw stem with constant preload on 5 screws of $8 \mathrm{kN}$, preload on screw 6 of $10 \mathrm{kN}$ 


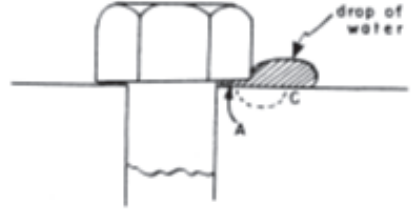

Fig. 4.: Interstitial corrosion (taken from Bickford (1995))

GBJ screws during reassembly of the joint and verification of the closing load. In fact, these tasks were not performed based on detailed procedures and with proper tools (e.g., torque wrench); rather, the operators were used to maintain the GBJ upon routine inspections, based on their experience. Maintenance actions on GBJ were neither tracked in maintenance reports, as GBJ was classified as not critical.

\subsection{Structural Reliability Model}

Given that the failed GBJ was a COTS, information about the characteristics of its materials was lacking and, as it emerges from Subsection 3.1 , possibly wrong. Then, the first phase of this step of the investigation was the characterization of the GBJ with respect to its geometry, materials and loads. For this, a CAD model of the joint was developed, and analyses were carried out based on the Finite Element Method (FEM). Consistently with the oucomes of the preliminary analysis (Subsection 3.1), we considered AISI 316 steel as screw material, with the following values of the yield and breakage strengths, respectively: $\sigma_{y}=450 \mathrm{MPa}, \sigma_{u}=700 \mathrm{MPa}$.

The analyses yielded the following results:

- Credible values of loads on the bolts are between $8 \mathrm{kN}$ and $12 \mathrm{kN}$, as also it results from a comparison with technical datasheets and experience.

- FEM analysis with non-linear materials showed that the asymmetry of the loads brings the screws into a stressful condition in the underhead area, consistently with what emerged from DMEA.

- Given the characteristics of the tank, we can assume that leakage cannot occurr when the pressure minimum value on the gasket is larger than $0.5 \mathrm{MPa}$.

- The total loss of tightening of a single bolt is not sufficient to have a leakage; this requires also a significant relaxation of at least one of the adjacent bolts.

Based on these findings, we first developed a creep model to simulate the loss of tightening, which was then combined with a crack corrosion model, encoding the two relevant mechanisms discussed in Subsection 3.3). We finally applied the Monte Carlo approach to consider the uncertainties on the FEM model parameters and propagate their effects onto the probability of leakage over time.

\subsubsection{Creep model}

There are few works (e.g., Kassner and Smith (2014); Kassner et al. (2015); Deibler (2014); Reed and Walsh (2017)) that model creep over a long time (i.e., dozens of years) of AISI 304/316 steel components operated in ground benign environments (i.e., room temperature, no significant vibrations, etc.). These propose the following two models:

$$
\begin{aligned}
& \dot{\epsilon}_{\text {creep }}=C_{1} \cdot \sigma^{C_{2}} \cdot t^{C_{3}} \\
& \epsilon_{\text {creep }}=a \cdot \sigma^{m} \cdot \log _{10} t
\end{aligned}
$$

where $\epsilon_{\text {creep }}$ represents the creep strain, whereas $\dot{\epsilon}_{\text {creep }}$ is its rate $[\mathrm{mm} /(\mathrm{mm} \cdot \mathrm{s})] ; \sigma$ is the stress level $[M P a], t$ is the time $[s]$, whereas $C_{1}, C_{2}$, $C_{3}, a$ and $m$ are constants of the models.

To estimate the parameters of the models in Eqs. $1-2$, a regression was performed on the literature data (Kassner and Smith (2014); Deibler (2014); Kassner et al. (2015)), which produced the estimates in Table 1.

Table 1.: Parameters of the models in Eqs. 1-2

\begin{tabular}{|cc|}
\hline parameter & value \\
$C_{1}$ & $2.84 \cdot 10^{-19}$ \\
$C_{2}$ & 5.72 \\
$C_{3}$ & -0.887 \\
$a$ & $4.14 \cdot 10^{-18}$ \\
$m$ & 5.62 \\
\hline
\end{tabular}

Figure 6 shows the average and minimum values over a time horizon of 25 years of the pressure on the teflon gasket for three different values of nominal preload (i.e., 8, 10 and $12 \mathrm{KN}$ ), equal for all six screws. The minimum pressure values always remain much higher than the threshold to have a leakage (i.e., 0.5 MPa). This means that the accident cause could not be just the creep phenomenon: if the GBJ bolt preloads had been correctly set and the GBJ properly operated, the leakage would not have occurred.

The two considered creep models produce very similar results, whereby the remaining part of the analysis was performed by relying on Eq. 1, only.

\subsubsection{Corrosion model}

From a careful review of the literature, it emerged that none of the available models of corrosion 


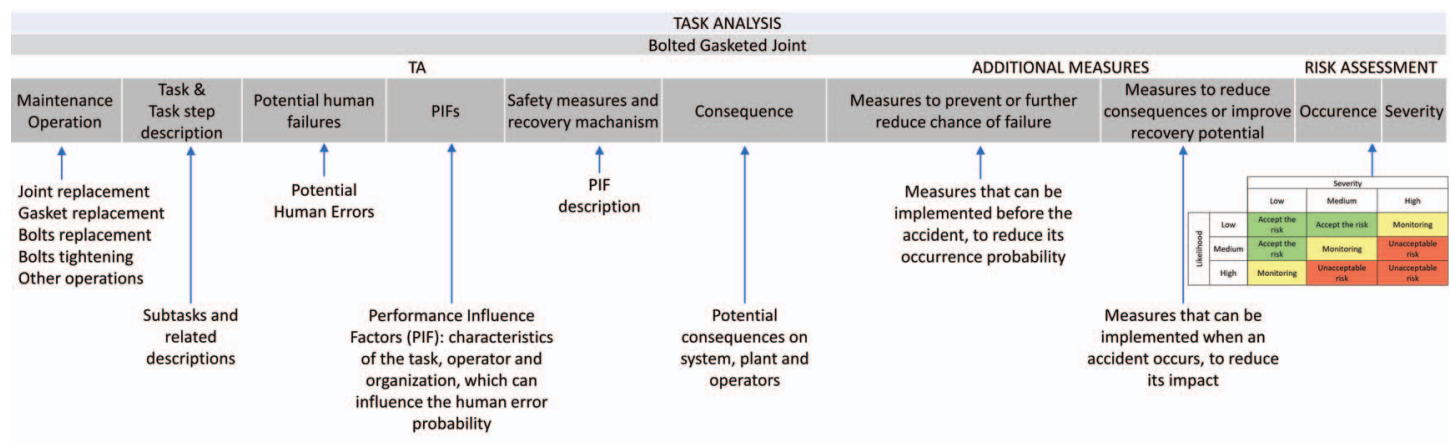

Fig. 5.: Worksheet for TA

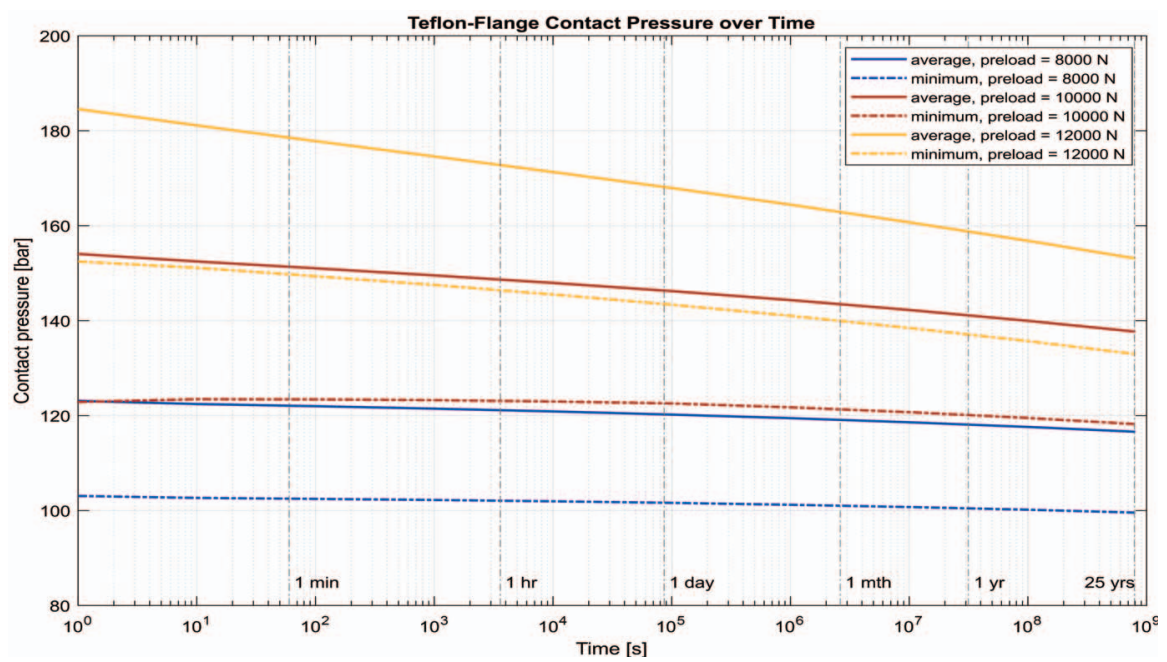

Fig. 6.: Pressure on the gasket

cracking can describe the crack propagation in case of small amplitudes of both thermal cycles and vibration and also small values of the GBJ internal pressure. Therefore, we used the FEM model to investigate whether and how the asymmetrical relaxation of the bolts due to creep can cause this phenomenon. Namely, with reference to Figure 7, we set to $15 \mathrm{KN}$ the preload values on screws $\mathrm{A}$ and $\mathrm{B}$, as this is large enough to generate the torsional overload damage underhead and the significant plastic deformation identified by the preliminary analysis, and to $10 \mathrm{KN}$ the preload on the remaining screws. An initial crack underhead was set on screw A.

Crack propagation was therefore encoded in the FEM model through the Cohesive Zone Model (CZM, Moslemim and Khoshravan (2015); Javidrad and Mashayekhy (2014)): the crack evolves (i.e., progressive elimination of the FEM nodes) once the energy absorbed in the fracture zone exceeds a critical value. In details, the stressseparation behavior in the area of the front of the crack was modeled through the following bilinear model (Figure 8), whose variables are specified in Table 2:

$$
\begin{aligned}
\sigma & =K_{n} \cdot u_{n} \cdot\left(1-d_{n}\right) \\
d_{n} & = \begin{cases}\frac{u_{n}-\bar{u}_{n}}{u_{n}} \cdot \frac{u_{n}^{c}}{u_{n}^{c}-\bar{u}_{n}} & u_{n}>\bar{u}_{n} \\
0 & \text { otherwise }\end{cases} \\
G_{c n} & =\sigma_{\max } \cdot u_{n}^{c} / 2
\end{aligned}
$$

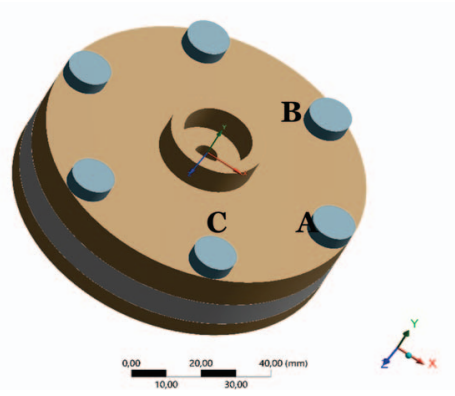

Fig. 7.: Screws A, B and C 


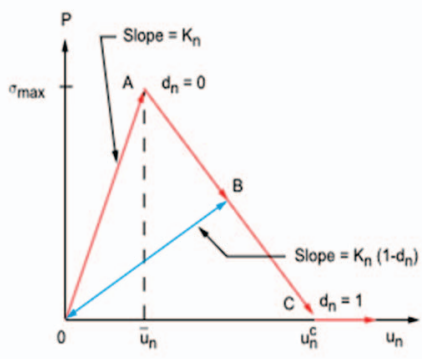

Fig. 8.: Corrosion-crack model

Table 2.: Variables of the model in Eq. 3

\begin{tabular}{|cc|}
\hline symbol & variable \\
\hline$\sigma$ & normal stress \\
$\sigma_{\max }$ & maximum allowable stress \\
$K_{n}$ & stiffness \\
$u_{n}$ & separation gap \\
$\bar{u}_{n}$ & separation gap at $\sigma_{\max }$ \\
$u_{n}^{c}$ & final separation gap \\
$d_{n}$ & separation parameter \\
$G_{c n}$ & critical fracture energy \\
\hline
\end{tabular}

In words, once the maximum allowable stress, $\sigma_{\max }$, is reached, the rigidity of the material progressively decreases until the complete detachment of the two surfaces $\left(u_{n}^{c}\right)$. The area underlying the arrrows in Figure 8 indicates the critical fracture energy, which was set to $G_{c n}=1.2 \cdot 10^{5}$. The FEM model showed that the progressive relaxation of screws $\mathrm{B}$ and $\mathrm{C}$ causes a corresponding increase in the load on screw A, which entails the gradual propagation of the crack underhead, given the stress concentration in the discontinuity area of the crack front. In the occurred accident, this phenomenon was accelerated by chloride stress corrosion cracking due to possible minimal leakages of hydrochloric acid vapors, gradually reducing the resistant section of the screw in a selfsustaining process that finally leads to the $\mathrm{rf}^{-}$ tively sudden cut of the screw head and to consequent loss of seal of the flange.

\subsubsection{Reliability model}

To find the probability of leakage over time, consider the epistemic uncertainty on the mo parameters (Table 3) and apply the Monte $\mathrm{C}$ c method for its propogation through the FI model. To reduce the computational burden sensitivity analysis was first carried out for creep model, to identify the parameters that $\mathrm{c}$ tribute most to the creep evolution. Figure $9 \mathrm{sc}$ marizes the results: $C_{2}$ has the greatest impact on the tightening force over the entire time horizon.

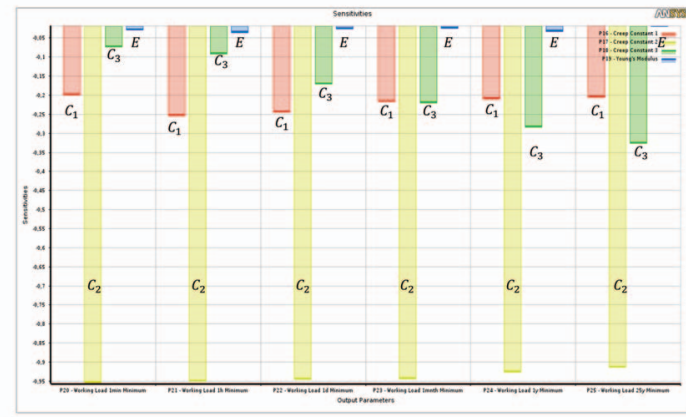

Fig. 9.: Sensitivity analysis: results relevant to different time instants

Then, we considered the uncertainty on $C_{2}$, only (Table 3).

With respect to CZM, instead, we considered the uncertainty on its most relevat parameter: $G_{c n}$ (Table 3).

Table 3.: Uncertainties on model parameters

\begin{tabular}{|c|c|c|}
\hline parameter & distribution & values \\
\hline \hline$C_{2}$ & Uniform & $U(5.72 / 2,5.72 * 1.5)$ \\
$G_{c n}$ & Normal & $N\left(1.2 \cdot 10^{5}, 4 \cdot 10^{4}\right)$ \\
\hline
\end{tabular}

The study showed that considering both degradation mechanisms (stress corrosion and relaxation), the probability of having an acid loss over the 25 years of system operation is in any case less than $4 \cdot 10^{-3}$ (Figures 10-11).

\subsection{Selection of safety barriers}

The barriers taken into consideration are the following:

- Barrier $B_{0}$ : use of a new type of bolts,

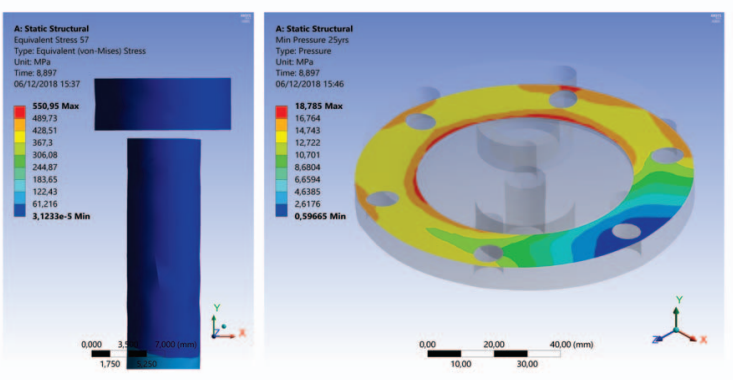

Fig. 10.: Corrosion-crak model 


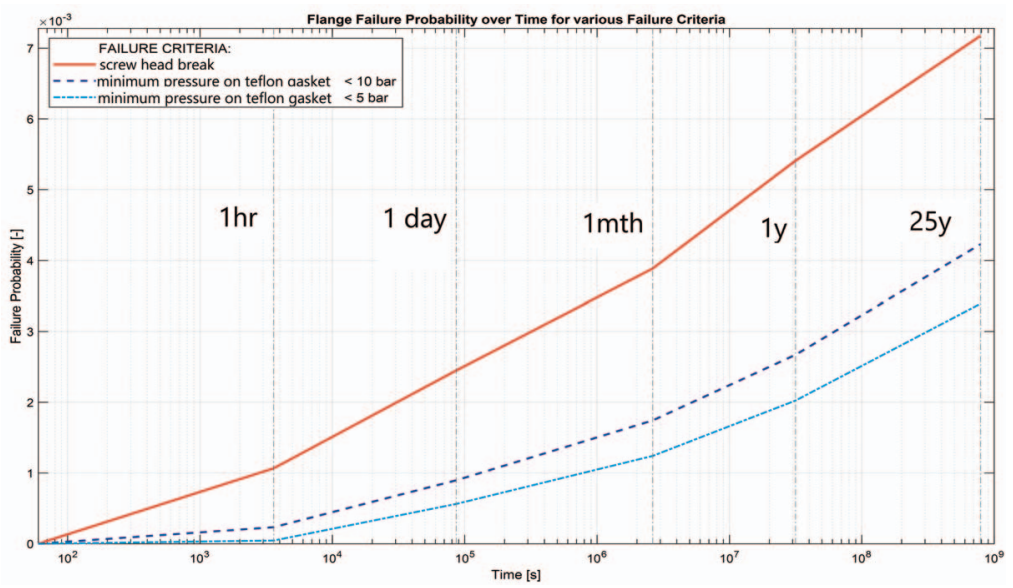

Fig. 11.: Corrosion-crak model

more suitable for working in the acid environment in which the GBJ operates. The bolts identified are Durostone EPR $\mathrm{S} 1$, for which various chemical resistance tests have been carried out.

- Barrier $B_{1}$ : installation in the WWTS area of two cameras, which can be brandished in all directions and equipped with zoom and night vision system.

- Barrier $B_{2}$ : installation of a multichannel control unit and four electrochemical detector-transmitters. This system isintegrated with the existing alarm systems.

- Barrier $B_{3}$ : installation of PVC pneumatic diaphragm valves under all reagent tanks of the WWTS. The valves are automatic and will close whenever $B_{2}$ detects acid vapors. In addition, they can be closed manually or by sending a command through the control system.

- Barrier $B_{4}$ : installation of two suction nozzles above the intake ports towards the other plants and four nozzles in the areas of the tanks. Construction of a collector for the conveyance of the flow from the nozzles to the aspirator and delivery to the abatement tower.

- Barrier $B_{5}$ : installation of 2 single-leaf fire doors with portholes, compliant with ISO 9001 product certifications.

To evaluate the effect of the barriers on the incidental event considered, the Bow-Tie technique (BT, CCPS (2018)) was used. This allows examininig the incidental scenarios, identifying their causes and possible evolutions, and highlighting the role of the barriers that can intervene to prevent and/or mitigate them.

The initiator event (IE) considered is the leakage of the joint (Figure 12), which can be prevented by the new type of bolts (barrier $B_{0}$ ).

Once the leakage occurrs, the detection system (barrier $B_{1}$ ) reveals the presence of acid vapors. The probability of success of this barrier in interrupting the evolution of the incidental scenario depends not only on its intrinsic reliability, but also on the sensitivity of the instrumentation and the stochastic process of dispersion of the acid vapor, which could be concentrated in areas different from those in which the detectors are positioned. Furthermore, the procedures used to manage the maintenance of the detectors are fundamental, to make them always able to detect vapors. The analysis of all these aspects is not reported in this work.

If barrier $B_{1}$ does not work, the acid leakage can be detected by the cameras (barrier $B_{2}$ ). The considerations above on the importance of the procedures to manage the barrier apply also $B_{2}$, concerning both the alarm signaling (procedures and operator training to identify the scenario) and the execution of the actions to be implemented to solve it.

If at least one of the detection barriers properly works (upper branch of the BT in Figure 12), the actuation of the valves (barrier $B_{3}$ ) blocks the evolution of the incidental scenario. The success of this barrier depends on the correct functioning of the control system and also on the possibility that the valves are activated manually. The human reliability analyses to check the effectiveness of the operator procedures are not reported in this work.

If the valves have not completely prevented the leakage, the captation system (barrier $B_{4}$ ) avoids the dispersion of acid vapors towards the adjacent plants. Notice that the possibility that there is an activation of this barrier independently on the other barriers is not considered. If this hypothesis is relaxed, there would be a further reduction in 
the probability of the accident scenarios. In this sense, the analysis carried out is conservative. The effect of the fire doors (barrier $B_{5}$ ) is to hinder the passage of acid vapors towards the adjacent plants. To be effective, this barrier must be always active, which entailed analysing the procedures to keep the doors closed. Moreover, we also checked through the analysis of dispersion of acid fumes that there are no other paths that steam can follow to go to neighboring plants. These analyses are not reported in this work.

Two incidental scenarios are therefore identified from the BT analysis (Figure 12): in scenario $S_{2}$, the undetected acid vapor moves towards the adjacent plants, whereas in scenario $S_{1}$ the presence of acid vapor is detected, providing the opportunity to make additional actions to manage the emergency. This allows us to consider other accessory mitigating actions to avoid damages outside the considered work environment.

Scenarios $S_{1}$ and $S_{2}$ depend on the failures of 5 and 4 barriers, respectively. The quantitative analysis of the BT is not reported in this work. However, even by a qualitative analysis, we can see that the probability of the sequence of events leading to the final effects of having acid vapors in adjacent plant is strongly reduced by the considered barriers.

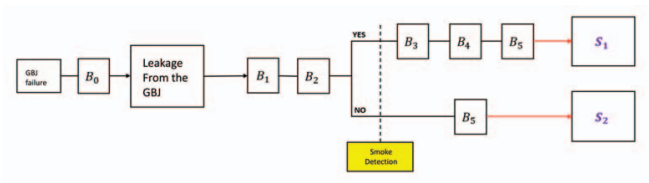

Fig. 12.: Bow-Tie model

\section{Conclusions}

In this work, the procedure followed to investigate an accident originated from a leakege in a GBJ has been showed. This is based on a combination of techniques and methodologies such as material analysis, structural reliabilty, atmospheric dispersion modeling and human reliability modeling. The study identified the causes of the leakage and proposed risk reduction measures, which reduce the probability of future accidents occurrence.

\section{References}

Abid, M. and D. Nash (2006). Joint relaxation behavior of gasketed bolted flanged pipe joint during assembly. In 2nd WSEAS International Conference on Applied And Theoretical Mechanics (MECHANICSO6) pp, pp. 319-325.

Bickford, J. (1995). An introduction to the design and behaviour of bolted joints, marcel decker inc., new york. Technical report, ISBN 0-82479297-1.
CCPS (1992). Guidelines for Investigating Chemical Process Incidents. Center for Chemical Process Safety of the American Institute of Chemical Engineers.

CCPS (2018). The Bow Tie Model, Chapter 2, pp. 15-52. John Wiley Sons, Ltd.

Deibler, L. A. (2014). Room temperature creep in metals and alloys. Technical report, Sandia National Lab.(SNL-NM), Albuquerque, NM (United States).

DOE (1999). Conducting Accident Investigations DOE Workbook, Revision 2. U.S. Dept. of Energy, Washington D.C, USA.

European Sealing Association (1998). Guidelines for safe seal usage-flanges and gaskets. Report No. ESA/FSA 9(98), 1-40.

HSE (accessed on Jan 2020). hiips://www.hse.gov.uk/humanfactors/topics/pifs.pdf.

ISO-60812 (2006). Analysis techniques for system reliability. Procedure for failure mode and effects analysis (FMEA).

Javidrad, F. and M. Mashayekhy (2014). A cohesive zone model for crack growth simulation in aisi 304 steel. Journal of Solid Mechanics 6, 378-388.

Kassner, M., K. Smith, and C. Campbell (2015). Low-temperature creep in pure metals and alloys. Journal of materials science 50(20), 6539-6551.

Kassner, M. E. and K. Smith (2014). Low temperature creep plasticity. Journal of materials research and technology 3(3), 280-288.

Khan, N. B., M. Abid, M. Jameel, and H. A. Wajid (2017). Joint strength of gasketed bolted pipe flange joint under combined internal pressure plus axial load with different (industrial and asme) bolt-up strategy. Proceedings of the Institution of Mechanical Engineers, Part E: Journal of Process Mechanical Engineering 231(3), 555-564.

Kirwan, B. and L. K. Ainsworth (1992). A guide to task analysis: the task analysis working group. Taylor Francis, Washington, D.C.

Moslemim, M. and M. Khoshravan (2015). Cohesive zone parameters selection for mode-i prediction of interfacial delamination. 61(9), $507+$.

PCC-ASME (2000). Guidelines for pressure boundary bolted flange joint assembly. American Society of Mechanical Engineers, NY, USA.

Reed, R. and R. Walsh (2017). Low-temperature creep of austenitic stainless steels. In Journal of Physics: Conference Series, Volume 897, pp. 012002. IOP Publishing.

Sklet, S. (2004). Comparison of some selected methods for accident investigation. Journal of Hazardous Materials 111(1), 29 - 37. A Selection of Papers from the JRC/ESReDA Seminar on Safety Investigation Accidents, Petten, The Netherlands, 12-13 May, 2003. 